\title{
A phase II RCT and economic analysis of three exercise delivery methods in men with prostate cancer on androgen deprivation therapy
}

Shabbir MH Alibhai ${ }^{1,2,8^{*}}$, Daniel Santa Mina ${ }^{1,3+}$, Paul Ritvo ${ }^{1,4 \dagger}$, Catherine Sabiston ${ }^{2 \dagger}$, Murray Krahn ${ }^{1,2}$, George Tomlinson ${ }^{1,2}$, Andrew Matthew ${ }^{1,2}$, Roanne Segal ${ }^{5}$, Padraig Warde ${ }^{1,2}$, Sara Durbano ${ }^{1}$, Meagan O’Neill ${ }^{1,2}$ and Nicole Culos-Reed ${ }^{6,7}$

\begin{abstract}
Background: Androgen deprivation therapy is commonly used to treat prostate cancer, the most common visceral cancer in men. However, various side effects often worsen physical functioning and reduce well-being among men on this treatment. Based on existing evidence, both resistance and aerobic training provide benefits for this population yet adherence rates are often low. The method of exercise delivery (supervised in-center or home-based) may be important, yet few studies have compared different models. Additionally, long-term exercise adherence is critical to achieve sustained benefits but long-term adherence data and predictors of adherence are lacking. The primary aim of this phase II, non-inferiority randomized controlled trial is to determine whether three exercise training delivery models are equivalent in terms of benefits in quality of life and physical fitness in this population. Secondary aims include examination of long-term adherence and cost-effectiveness.
\end{abstract}

Design: Men diagnosed with prostate cancer, starting or continuing on androgen deprivation therapy for at least 6 months, fluent in English, and living close to one of two experienced Canadian study centers are eligible. Participants complete five assessments over one year, including a fitness assessment and self-report questionnaires. Socio-demographic and clinical data collection occur at baseline, bone mineral density testing at two time points, and blood work is performed at three time points. Participants are randomized in a 1:1:1 fashion to supervised personal training, supervised group training, or home-based smartphone- and health coach-supported training. Each participant receives a detailed exercise manual, including illustrations of exercises and safety precautions. Participants are asked to complete 4 to 5 exercise sessions per week, incorporating aerobic, resistance and flexibility training. Participant intensity levels will be monitored. The intervention duration is 6 months, with 6 months additional follow-up. Outcomes include: body composition, fitness testing, quality of life and fatigue, biological outcomes, and program adherence. Cost information will be obtained using patient diary-based self-report.

Discussion: The goals of this study are to gain a better understanding of health benefits and costs associated with commonly used yet currently not compared exercise delivery models as well as an increased understanding of adherence to exercise.

Trial registration: The trial has been registered at clinicaltrials.gov (Registration \# NCT02046837), registered January $20^{\text {th }}, 2014$.

Keywords: Prostate cancer, Androgen deprivation therapy, Exercise, Randomized controlled trial, Quality of life, Fatigue, Physical fitness, Cost-effectiveness, Patient adherence

\footnotetext{
*Correspondence: shabbir.alibhai@uhn.ca

${ }^{\dagger}$ Equal contributors

University Health Network, Toronto, ON M5G 2C4, Canada

${ }^{2}$ University of Toronto, Toronto, ON M5S 2J7, Canada

Full list of author information is available at the end of the article
} 


\section{Background}

Prostate Cancer (PC) is the leading cancer diagnosed in North American men [1,2]. With improvements in early diagnosis and treatment, survival has been steadily increasing for this population [2]. One common treatment for men diagnosed with $\mathrm{PC}$ is androgen deprivation therapy (ADT), which is estimated to be used in $45 \%$ of men with PC [3,4]. Common side effects from ADT are fatigue, decreased muscle strength and cardiorespiratory function, loss of sexual functioning, mood changes, changes in body composition and negative effects on health-related quality of life (QOL) [5-8]. These changes often lead to a decrease in overall physical functioning and individual well-being $[6,9]$. Modifiable factors that can limit the burden of ADT need to be identified.

Exercise in the form of both resistance and aerobic training provide benefits for individuals with PC on ADT. In a randomized controlled trial (RCT), Segal et al. [10] found improvements in fatigue in the exercise group compared to a worsening of fatigue in the control group. Improvements in QOL were also noted following this incenter, structured exercise program [10]. Galvao et al. [11] found improvements in muscle strength following a 20week resistance and endurance training program [11]. Improvements in 6-minute walk tests, stair climbing and chair rise fitness measures were also observed. In another study, Galvao et al. found significant improvements in the general health and physical health composite scores on the Medical Outcomes Study 36-item questionnaire (SF36) for participants who completed a 12-week aerobic and resistance exercise program, compared to usual care [12].

These trials and others have been included in multiple systematic reviews of exercise interventions for individuals on ADT for PC. These reviews have found that, generally, exercise is safe and feasible, and exercise interventions are associated with multiple benefits for this population [9,13-16]. For example, Baumann et al. concluded that exercise interventions led to improvements in fatigue levels, muscular strength, flexibility, aerobic fitness, QOL, and well-being [9]. Santa Mina et al's review also found similar results regarding physical functioning and QOL outcomes with the additional finding that exercise program supervision by a certified professional was an important factor in achieving greater health benefits [14]. Altogether, findings from these reviews provide strong evidence of exercise benefits for patients on ADT.

Of interest, Thorsen et al. noted that in two of the studies reviewed, older age was associated with lower levels of participation intention [13]. This may be particularly important since PC is predominantly an older person's disease, and aging itself is associated with multiple functional declines and sarcopenia [17].

While the most consistent benefits of exercise interventions in men on ADT have been seen with 1:1 supervised programs [15], few studies have directly compared exercise delivery methods. The systematic review by Keogh et al. noted significantly greater improvements in outcomes (physical functioning and QOL) for individuals in group-based supervised training vs. home-based training [15]. However, three important things should be noted. First, the noted improvement with group-based training compared to home-based training was based on indirect study comparisons. Second, home-based training provided significant pre-post increases in aerobic endurance and reduced fatigue. Third, only one RCT has directly compared multiple exercise delivery methods, comparing only 1:1 and group supervised training, but with a small sample size and without a home-based arm [18]. Because in-center, supervised, exercise programs are resourceintensive (and largely inaccessible), the demonstration of similar efficacy in home-based programs to these supervised programs would have important health service delivery implications and economic benefits and may help expand exercise delivery options for men on ADT.

It is also clear that the benefits obtained from an exercise program are maintained only as long as the exercise is maintained; therefore ensuring long term adherence to exercise programming has significant health implications. In RCTs of exercise in PC, adherence responses to exercise interventions are moderate to high but vary by protocol design. Supervised exercise intervention adherence ranged from $79 \%$ to $95 \%$ (as assessed by attendance at scheduled sessions) in three studies $[10,19,20]$, whereas in a study of group-based training the attendance-based adherence was $94 \%[11,12]$. In three home-based studies, the adherence ranged from $77 \%$ to $100 \%$ (assessed by way of patient reporting, booster session attendance or weekly group session attendance) [21-23]. In spite of a moderate to high level of adherence for exercise intervention across multiple delivery approaches, only Culos-Reed et al. [21] directly measured changes in activity levels (decreased in strenuous physical activity, however mild and moderate physical activity stayed relatively the same) from postintervention to subsequent follow-up assessment. Additionally, follow-up assessments of fitness outcomes following the post intervention assessment [20-23] are rarely reported. However, when reported longer-term benefits vary, such that there is evidence of maintenance of intervention-based improvements at 6 months follow up in one study [20] whereas no differences in fatigue levels were found from baseline to the end of a 4-week intervention and again 4 weeks later, between the control and exercise groups [23].Follow-up data post-intervention were not obtained or reported in four of the above studies $[10,12,19,22]$.

Even though multiple rigorous studies have demonstrated improvements in several health outcomes with an exercise intervention, exercise in the oncology setting 
remains unfunded in most countries by health insurance programs (public and private) and organizations. This contrasts sharply with cardiovascular rehabilitation, which is publicly funded in many jurisdictions, in part due to more advanced, robust evidence demonstrating costeffectiveness. Indeed, in today's fiscally strained funding environment, there is an increasing emphasis on demonstrating value (or return) on investment. Demonstration of the cost-effectiveness of exercise programs for men on ADT could lead to larger scale implementation of these programs.

Thus, the primary aims of this phase II non-inferiority RCT are:

- To determine the feasibility of conducting a large multi-center non-inferiority RCT of three exercise delivery models in men with PC on ADT. Feasibility outcomes include participant recruitment, retention, adherence, outcome capture, and satisfaction;

- To obtain preliminary efficacy estimates for (a) group-supervised in-centre, and (b) home-based (smartphone-assisted) supported exercise programs, compared to (c) a 1:1 supervised in-center exercise program study for the clinical outcomes of QOL and physical fitness, and to select a primary outcome for a subsequent phase III trial;

- To examine adherence to exercise and predictors of adherence in each exercise group during the 6-month intervention and for 6 months after program completion;

- To determine the feasibility of conducting an economic analysis comparing exercise interventions and usual care.

\section{Methods}

This trial is taking place at two experienced academic tertiary care Canadian centers - the Princess Margaret Cancer Centre (Toronto, Ontario), and the Tom Baker Cancer Centre, (Calgary, Alberta). Ethics approval has been obtained at both institutions. All study participants provide written informed consent prior to study enrolment. The trial has been registered at clinicaltrials.gov (Registration \# NCT02046837).

\section{Study population/participants}

Men diagnosed with histologically confirmed PC of any stage, starting or continuing on ADT for at least 6 months (or who remain biochemically castrate after stopping ADT), able to speak, read and write in English, and living in close proximity to either study center are eligible for the study. Each potential participant is screened with the Physical Activity Readiness questionnaire (PAR-Q+ or PARmed-X) [24] and/or receives physician approval. If an individual already meets the American College of Sports
Medicine (ACSM) guidelines for moderate to vigorous physical activity (MVPA) or has a condition that would interfere with his ability to participate (e.g. severe arthritis limiting ambulation, major neuropsychiatric abnormality, severe visual/hearing loss, poorly controlled pain), he is excluded from the study.

\section{Recruitment}

The research coordinator at each study site will oversee recruitment. Estimated time for the recruitment phase will be 9 months, with the aim of recruiting 100 participants total. Potential participants are screened through PC clinics (primarily urology or radiation oncology) at each site and approached based on initial medical record review by the research coordinator. Potential participants are provided with a brief description of the study, and given an opportunity to ask any questions. If they are interested in study participation, written informed consent is obtained and a baseline assessment will be scheduled.

\section{Assessments}

Participants complete five assessments in total: at baseline, three months, six months (end of intervention), nine months, and twelve months. The nine- and twelvemonth assessments provide follow-up information after completion of the active intervention. Blinded outcome assessments are conducted by a Certified Exercise Physiologist (CEP; Canadian Society of Exercise Physiology, CSEP) or Registered Kinesiologist.

\section{Baseline assessment}

The baseline assessment consists of obtaining sociodemographic and clinical data as well as assessing fitness using measures of resting heart rate (HR), blood pressure (BP), oxygen saturation, body composition (body mass index, waist circumference and body fat percentage), upper-lower body strength testing, and cardiovascular testing (peak VO2). Participants also complete questionnaires (see below) and are scheduled for a bone mineral density (BMD) test (if one has not been completed in the last year) and blood work (fasting glucose, prostatespecific antigen (PSA), testosterone, hemoglobin, and cholesterol profile). Serum is also banked for ancillary studies on participants at the Toronto site. All measures are detailed below and summarized in Table 1 .

\section{Assessments (three, six, nine and twelve months)}

All follow-up assessments include updates on clinical data, fitness measurements, and self-report questionnaires. BMD testing occurs only at baseline and twelve months, blood work and body composition testing is done only at baseline, six months and twelve-months (Table 1). 
Table 1 Summary of study measures at specified time points

\begin{tabular}{|c|c|c|c|c|c|c|}
\hline Domain/Measure & Time required & $\mathrm{T}_{0}$ : (Baseline) & $T_{1}:(3 \mathrm{mo})$. & $\begin{array}{l}\mathrm{T}_{2}: 6 \mathrm{mo} . \\
\text { (End Int.) }\end{array}$ & $\begin{array}{l}\mathrm{T}_{3}: 9 \mathrm{mo} . \\
(3 \mathrm{mo} . \mathrm{f} / \mathrm{u})\end{array}$ & $\begin{array}{l}\mathrm{T}_{4}: 12 \mathrm{mo} . \\
(6 \mathrm{mo} . \mathrm{f} / \mathrm{u})\end{array}$ \\
\hline \multicolumn{7}{|l|}{ Quality of life } \\
\hline FACT-G (primary) & $8-10 \mathrm{~min}$ & $\bullet$ & $\bullet$ & $\bullet$ & $\bullet$ & $\bullet$ \\
\hline FACT-P & 4-5 min & $\bullet$ & $\bullet$ & $\bullet$ & $\bullet$ & $\bullet$ \\
\hline FACT-F & $5 \min$ & $\bullet$ & $\bullet$ & $\bullet$ & $\bullet$ & $\bullet$ \\
\hline \multicolumn{7}{|l|}{ Physical Fitness } \\
\hline $\mathrm{VO}_{2}$ Peak & $20 \mathrm{~min}$ & $\bullet$ & $\bullet$ & $\bullet$ & $\bullet$ & $\bullet$ \\
\hline Sit-to-Stand test & $1 \mathrm{~min}$ & $\bullet$ & $\bullet$ & $\bullet$ & $\bullet$ & $\bullet$ \\
\hline Grip Strength & $1 \mathrm{~min}$ & $\bullet$ & $\bullet$ & $\bullet$ & $\bullet$ & $\bullet$ \\
\hline Biological Outcomes & $<5 \min$ & $\bullet$ & & $\bullet$ & & $\bullet$ \\
\hline
\end{tabular}

Blood glucose

Cholesterol profile

PSA (safety)

Testosterone

Hemoglobin (covariate)

Body Composition

Bone mineral density

Body composition\$

\section{$30 \mathrm{~min}^{*}$ \\ $5 \mathrm{~min}$}

Adherence

Accelerometer

GLTEQ

$<5 \min$

Sessional attendance\#

-

Adherence Predictor Variables

$\begin{array}{ll}\text { NEWS-A } & 10 \mathrm{~min} \\ \text { HCCQ } & 5 \mathrm{~min} \\ \text { BREQ2 } & 5 \mathrm{~min} \\ \text { ROPAS } & 5 \mathrm{~min} \\ \text { PAB questionnaire } & 5 \mathrm{~min} \\ \text { Sedentary Behaviours } & 5 \mathrm{~min}\end{array}$

Cost-Effectiveness

Health questionnaire $\quad 5 \mathrm{~min}$

EQ-5D

5 min

Study Completion

5 min

$\begin{array}{ll}5 \text { min } & \bullet \\ \text { min } & \bullet \\ \min & \bullet \\ \min & \bullet\end{array}$

*Can be done on separate day to reduce participant burden.

\$Includes waist circumference, waist circumference:height ratio, and \% body fat using bioelectrical impedance analysis.

\#Only for those in supervised groups (done weekly).

Abbreviations: BREQ2 Behavioral Regulations in Exercise Questionnaire - 2, EQ-5D EuroQol 5 dimensions of health scale, FACT-G Functional Assessment of Cancer Therapy General, FACT-F Fatigue subscale, FACT-P Prostate subscale, GLTEO Godin Leisure Time Exercise Questionnaire, HCCQ Health Care Climate Questionnaire, Int Intervention, NEWS-A Neighborhood Environment Walkability Scale short form, PAB Planning, Attitudes, \& Behavior questionnaire, PSA prostate-specific antigen, ROPAS Relatedness to Others in Physical Activity scale, VO2 Peak Peak Volume of Oxygen Consumption.

\section{Randomization}

Participants are allocated to treatment groups following the baseline assessment through a computer-generated stratified randomization scheme developed by the study biostatistician (GT) and administered through an independent website. Although the CEP outcome assessors are blinded to treatment assignment, for practical reasons participants and the intervention CEPs cannot be blinded. Participants are randomized equally to one of three groups: 1:1 supervised training, supervised group training, and home-based smartphone-assisted training. Randomization is stratified by duration of prior ADT 
use ( $<3$ months versus 3 months or more), as evidence suggests that muscle changes and impact on QOL is most appreciated within the first 3-6 months of ADT use [6] and exercise response may be partially modified by duration of ADT [25].

Following randomization, participants are scheduled to attend their first session (1:1 or group-based training) or an orientation session (delivered by the study coordinator) for home-based training. All participants will receive a study manual at this time point.

\section{Objective measures/ Primary and secondary outcomes Body composition}

Body composition is measured following the CSEP's [26] Canadian Physical Activity Training for Health [27] assessing waist circumference (WC), weight, waist to hip ratio (WHR), body mass index (BMI). Body fat percentage $(\mathrm{BF} \%)$ is measured via bioelectrical impedance analysis (BIA) using the Tanita TBF-300A device (Illinois, USA). Additionally, participants receive a BMD scan of the lumbar spine, total hip, and femoral neck using dual $\mathrm{x}$-ray absorptiometry.

\section{Fitness testing}

Prior to the start of fitness testing, resting HR, BP, and oxygen saturation are measured. Aerobic fitness is directly assessed through the modified Bruce treadmill protocol (treadmill-based graded exercise test) [28], which assesses volitional peak oxygen consumption $\left(\mathrm{VO}_{2}\right.$ peak) using a metabolic cart (Parvo Medics TrueOne 2400 Metabolic Measurement System). Lower body strength is measured with a one minute sit-to-stand test $[29,30]$ and grip strength is used to assess upper body strength. Grip strength is measured with a Jamar dynamometer averaging three readings obtained in each hand [31].

\section{QOL and fatigue}

Functional Assessment of Cancer Therapy - General (FACT-G) is used to assess general health-related QOL [32], whereas the FACT-P (Functional Assessment of Cancer Therapy - Prostate) evaluates prostate-specific QOL [33] and the FACT-F (Functional Assessment of Cancer Therapy - Fatigue) is used to evaluate cancer-related fatigue [34]. All three questionnaires are validated and have been widely used in cancer research measures, and in particular, in RCTs of exercise in men on ADT [14].

\section{Biological outcomes (blood data collection)}

At three assessment time points (baseline, 6 months and 12 months), participants' blood is drawn to analyze fasting blood glucose, lipid profile (total cholesterol, low density lipoprotein, high density lipoprotein, and triglycerides), testosterone, as well as PSA levels. Blood glucose and lipids are being monitored as ADT is associated with an increased risk of both diabetes and dyslipidemia $[35,36]$; conversely, exercise is associated with improvements in both metabolic parameters [37]. PSA levels are being monitored as a safety measure [38], as in other exercise trials $[10,12,39]$, and testosterone is measured to assess adequacy of castration.

\section{Adherence}

Program adherence and predictors of adherence are assessed. Adherence measures include physical fitness outcomes (as the most proximal, objective outcome of exercise adherence), accelerometry, as well as attendance at exercise sessions (for those in supervised programs). For those in the home-based, smartphone-assisted arms, use of the selfreport software (Connected Wellness Platform, NexJ Systems, Toronto, Canada) and interview responses (obtained by health coaches) will provide proxies for attendance and exercise program engagement. Accelerometers (Actigraph GT3X, Pensacola, FL) will be worn for seven days at each assessment time point. Accelerometers will be worn during waking hours, allowing for capture of total physical activity during the observation period [40]. Data will be extracted from the accelerometer in 60-second epochs and will be screened through; (i) at least 4 days of valid data, including (ii) at least 10 hours of wear time per day; (iii) non-wear time will be assessed as periods of time with no movement ( 0 counts per minute) for more than one hour at a time.

Two common definitions of adherence are adopted: 1) at least 150 minutes of MVPA per week, based on the ACSM guideline for cancer survivors [28] and the revised CSEP guideline for adults and older adults [41]; and 2) at least 10,000 steps per day [42] for $6+$ days per week. In a sensitivity analysis, a recently described age-specific cutoff of 7,500 steps per day for older adults [43] is also tested. The accelerometer data will be analyzed by examining the total amount of MVPA and sedentary behavior during the 7 days. MVPA is defined as activity $>1952$ counts per minute [42] and sedentary behaviour as $<100$ counts per minute and time spent inclined.

Predictors of adherence are targeted based on a social ecological framework (Figure 1) [44]. Multiple potential determinants of exercise adherence and the interrelatedness of these determinants are examined using brief validated measures at each level: exosystem (Neighborhood Environment Walkability Scale: Short Form (NEWS) [45]); mesosystem (Health Care Climate Questionnaire [46] and Related to Others in Physical Activity Scale [47]); and microsystem (Behavioral Regulations in Exercise Questionnaire-2 [48,49] and a Planning, Attitudes, \& Barriers scale [50,51]).

\section{Intervention}

The six-month exercise intervention consists of one of three exercise delivery arms; supervised 1:1 personal 


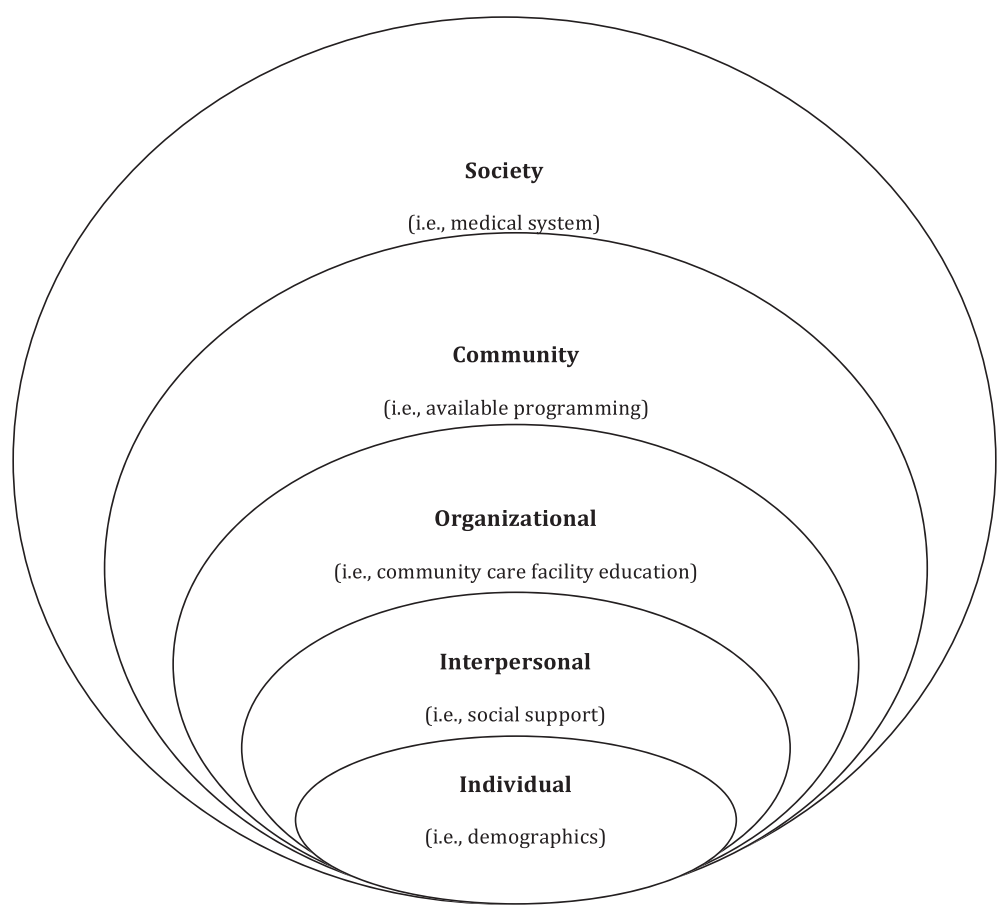

Figure 1 Social ecological framework for understanding exercise determinants.

training, supervised group training and unsupervised home based (smartphone-assisted) training. In all three intervention arms, each participant meets with a CEP to receive instructions regarding the exercise program and orientation to a detailed exercise manual that includes illustrations of exercises and safety precautions for exercising. All participants are asked to complete 4-5 days per week of mixed modality exercise incorporating aerobic, resistance and flexibility training, all training programs are prescribed based on the FITT principle: Frequency, Intensity, Time and Type. Each program includes an education component of 12 topics that will focus on common concerns facing new exercisers (see Table 2). This occurs during their sessions or weekly phone calls throughout the intervention period, administered by the CEP for the 1:1 supervised and groupbased training, and by health coaches for home-based participants. Both group and 1:1 supervised training occur under the supervision of a CEP. Participants' programs are individualized based on their baseline fitness assessment, with the target time and absolute workload (target heart rate: $60-70 \%$ of heart rate reserve) kept the same across all interventions. Relative intensity is maintained throughout the program; therefore, participants are progressed similarly to ensure this occurs (see Progression section below).

The 10-point Rating of Perceived Exertion (RPE) scale is used to monitor exercise intensity levels [52], with participants instructed to maintain their intensity level at 3-6 during exercise sessions. RPE levels are related to target HR, as it corresponds to the linear change in HR [53]. HR monitors (Polar, NY, USA) are used at 3-week intervals during the exercise sessions across all intervention arms. This is to ensure participants maintain appropriate heart rate levels during the session, ensuring calibration with the 3-6 rating on the RPE scale throughout the intervention period. Home-based participants are trained to use HR monitors which are provided to them. Participants in the supervised 1:1 and group programs will have staff present to assist in HR measurement.

Study staff will hold monthly conference calls and staff will be trained on testing and training procedures, to ensure standardization across both sites. Supervised exercise sessions are documented with standard forms at all sites.

\section{Progression}

Participant progression is individualized, and monitored by a CEP or health coach every three weeks to ensure that progression is occurring. The participant's intensity level during the exercises sessions (both aerobic and resistance) will be used as an indicator of whether the participant is ready to progress. CEPs or health coaches adjust intensity levels to ensure participants maintain the desired intensity range throughout each exercise session. Documentation of this progression is completed using a standard form across all arms of the intervention and both study sites. Participants who are working below their target HR when performing aerobic exercise will 
Table 2 Education topics

\begin{tabular}{|c|c|}
\hline Education topics & Key points \\
\hline \multirow[t]{3}{*}{ 1) Introduction to Exercise } & - Benefits of Physical Activity \\
\hline & - Program targets 3 areas of PA (aerobic, resistance, and flexibility) \\
\hline & - PA is safe, feasible and has shown to provide benefits \\
\hline \multirow[t]{4}{*}{ 2) Goal Setting } & - Goal setting will assist with your dedication and motivation to complete the exercises \\
\hline & - SMART Goals - Specific, Measurable, Attainable, Realistic, Timely \\
\hline & - Use the goal worksheet in the manual \\
\hline & - Make long term and short term goals \\
\hline \multirow[t]{3}{*}{ 3) Behavior Change } & - The plan you set out may not be followed $100 \%$ \\
\hline & $\begin{array}{l}\text { - Anticipate obstacles that may come as you are changing a behaviour and develop strategies for } \\
\text { dealing with it before it arises }\end{array}$ \\
\hline & - Monitor your progress, Reward yourself, Visualize your success \\
\hline \multirow[t]{3}{*}{ 4) Planning for Barriers } & - Biggest perceived obstacles \\
\hline & - Lack of time, self-discipline, partner and ability \\
\hline & - Plan ahead for periods of inactivity \\
\hline \multirow[t]{2}{*}{ 5) Social Support } & - You are more likely to be successful if your family, friends and even co-workers are supportive of you \\
\hline & - Social support can occur in many forms - encouragement, completing activities with you, etc. \\
\hline \multirow[t]{3}{*}{ 6) Monitoring Behavior } & - Mix up your activities to stay motivated \\
\hline & - Try something new, or something you have done previously \\
\hline & - It is very easy to enter an exercise rut \\
\hline \multirow[t]{3}{*}{ 7) Maintaining Motivation } & $\begin{array}{l}\text { - Greatest source of motivation: Fun/enjoyment/stimulation, feeling of accomplishment, pleasure of } \\
\text { learning and benefits (i.e. improved sleeping) }\end{array}$ \\
\hline & - Pursue something that you enjoy, that is convenient to your schedule \\
\hline & - Take opportunities to be active \\
\hline 8) Personal Control & $\begin{array}{l}\text { - Believing that you are in control of your own life give you reinforced motivation and further } \\
\text { commitment to make changes }\end{array}$ \\
\hline \multirow[t]{3}{*}{ 9) Self- Discipline, Reward \& Attitude } & - Self-discipline can result in increased productivity, improved self-esteem and confidence \\
\hline & - Rewards - use workbook in manual \\
\hline & - Attitudes toward change can determine whether you will be successful \\
\hline 10) Adapting your Program & - Adapting your program - FITT principle \\
\hline 11) Health and the Media & $\begin{array}{l}\text { - Be mindful of the 'Get fit quick' media marketing - Health eating and regular PA will help maintain a } \\
\text { long-term health lifestyle }\end{array}$ \\
\hline \multirow[t]{2}{*}{ 12) Lifelong Active Living } & - Use some of the tips and tricks in the manual to assist with continuing your active life \\
\hline & - Change things up, work towards small goals, work with a friend, etc. \\
\hline
\end{tabular}

first increase their exercise duration (i.e., from 15 to 20 minutes), followed by an increase in exercise intensity (i.e., increase walking speed or grade). Similarly, participants who are able to complete $\geq 12$ repetitions and 3 sets of a specific resistance exercise will increase the resistance (i.e., blue band to purple band; next resistance level) they are performing the exercise at to ensure that they are continuing to progress effectively throughout the program.

\section{1:1 supervised training}

Participants in the 1:1 supervised training complete three sessions per week with a CEP for a period of six months, and are encouraged to perform one to two additional weekly independent (home-based) sessions. Each exercise session consists of cardiovascular training for 15 to 30 minutes, resistance training exercises (working major muscle groups), and flexibility training (including 5-10 minutes of stretching at the end of each session). All participants are provided with resistance bands for home use.

\section{Group supervised training}

This protocol differs from the 1:1 training protocol in only one way: a group of 4-6 individuals are supervised by a CEP at each session. 


\section{Home-based training}

The same protocol and training frequency as the 1:1 and group supervised training program is explained and recommended to be followed by the home-based smartphone-assisted participants. Along with the resistance bands provided, participants in the homebased training group also receive a stability ball, an exercise mat, a HR monitor (with instructions), and a smartphone with a 6-month paid phone and data plan. Participants in the home-based arm complete an orientation session with a CEP prior to the commencement of the training. Participants are oriented to the same exercise manual that is provided to the supervised group participants, and review the resistance and flexibility exercises with a CEP to ensure proper technique. During the orientation participants also meet with the health coach to review the smartphone application and the role of the health coach. Remote health coaches and smartphone technology are intended to provide support to participants in the home-based training program during the intervention phase, as there is no direct (i.e. face-to-face) supervision with this group. The health coach connects with participants on a regular basis (ideally once each week) to follow up on the week's exercise sessions and to provide guidance for the participants and assist with any smartphone application issues. Health coaches instruct the participant when to use the HR monitors to evaluate intensity every three weeks. A customized smartphone application is used (Connected Wellness Platform) that allows users to input health information, levels of symptoms, and exercise routines, as well as tracking progress over time.

\section{Tapering}

\section{Supervised sessions}

Tapering allows participants within the supervised training arms to transition from predominately supervised training to independent exercise training. This is in place to encourage participants to continue to exercise independently at the same intensity and frequency as achieved during their supervised exercise programs. Tapering occurs for participants in the 1:1 supervised and groupbased training arms. Starting in month five, supervised sessions are reduced to two sessions per week. In the final month (month six), supervised sessions are tapered further to one session per week. Participants are encouraged to replace the supervised sessions with equivalent independent exercise sessions.

\section{Health coach support}

For the home-based group, a similar concept of tapering will be introduced to reduce the level of support provided by health coaches. In the first four months the health coaches will contact each participant once every week and will be available seven days per week, with turnaround time within an hour. In the final two months of the intervention, the health coaches will contact each participant once every two weeks and will be available five days a week. Text message turnaround time will be reduced from within the hour to next day.

\section{Safety}

The CEP reviews with each participant precautions and safety while exercising during the participant manual orientation and participants are reminded to review the manual prior to commencing independent exercise sessions. All exercise trainers have cardiopulmonary resuscitation (CPR) and automated external defibrillator (AED) training and have received safety training at each site. Any adverse events are document using the National Cancer Institute common terminology criteria for adverse events v4.0 [54].

\section{Cost-effectiveness analysis}

This phase II study investigates the feasibility of completing a phase III trial and a full companion economic evaluation. In the phase II design there will be no formal cost-utility analysis, however outcomes relevant to economic analyses regarding health status will be collected at each assessment time point using a generic utility instrument (the European QOL 5-dimension measure (EQ5D)) [55]. Costs are captured with a patient-reported diary that has been used in prior studies of men with PC $[56,57]$. Costs related to health care utilization (e.g. visits to a physician or physiotherapist), transportation, purchase of exercise equipment (including gym membership fees), and related costs are captured at each of the five time points where QOL and fitness data are captured.

\section{Sample size calculation/power}

Following standard guidelines for a phase II RCT [58-60], we have targeted 30 patients per arm (90 patients in total) to provide precise estimates of parameters related to the primary outcomes as well as important feasibility information that will be crucial to inform the phase III study. Assuming 10\% drop-out, we will recruit 100 patients across both sites.

\section{Statistical analysis}

Statistical analysis focuses on feasibility outcomes and those which will inform decisions about moving forward to and planning a phase III clinical trial. Feasibility outcomes include recruitment rates, and proportions of participants retained and adherent at landmark times throughout the study, all of which will be estimated with 95\% confidence intervals. Additionally tallies are kept on reasons for not participating. Each participant also completes an exit interview and satisfaction survey which 
will be analyzed for participant satisfaction within the study and cost effectiveness analysis.

Adherence is assessed through the two different binary definitions given previously, based on steps or hours of exercise. We estimate proportions adhering to exercise in each three-month period and use generalized estimating equation logistic regression models to compare adherence between groups and assess predictors of adherence over the course of the study. Predictors include sociodemographic (e.g. age, education), clinical (disease stage), baseline fitness, and ecological variables (NEWS, etc.).

Quality of Life (FACT-G and FACT-P), musculoskeletal fitness, and aerobic fitness are efficacy outcomes that are analyzed using linear mixed effects model with subject-specific random effects and group-by-time interactions. The analysis will not focus on differences between groups, but, to assist with phase III trial design, estimation of measures of within and between-person variance.

As previously mentioned, there is no formal costeffectiveness analysis, however data obtained (diary and EQ-5D completion and participant acceptance) is used to help develop this outcome for the phase III study. Descriptive estimates of costs by category and per intervention arm as well as per site (Toronto, Calgary) will be calculated.

\section{Criteria to move forward to phase III RCT}

To move forward to the phase III intervention, we have identified pre-specified criteria which must be met during the phase II study. Reasonable recruitment (at least $25 \%$ of potentially eligible approached participants), adherence $(70 \%)$ and retention rates (70\%), at least moderate satisfaction of participants, and capture of at least $80 \%$ of clinical outcomes collected are the minimum criteria. The intention is that all three of the interventions will move forward to the phase III study; however, if one arm proves to be inferior to the other two, based on adherence, retention, satisfaction or QOL and physical fitness results, then only two will move to phase III.

\section{Discussion}

Increasing amounts of data from well-designed intervention studies have demonstrated statistically and clinically significant gains in fitness and QOL outcomes with exercise-based interventions [16]. The most robust data support 1:1 supervised programs, but these are resource-intensive and largely inaccessible to most patients. Whether group-supervised or home-based programs, both of which are likely significantly less resourceintensive and more feasible to implement, are equally effective as 1:1 supervised programs remains unclear. Altogether, wider adoption of exercise programs is important as the status quo of the majority of men treated with $\mathrm{ADT}$ is that they remain physically inactive and at risk of multiple potentially avoidable side effects $[13,61,62]$. In addition, longer-term adherence, after completion of acute training programs, is key to ensuring ongoing health benefits, yet few data have been published examining longer-term follow-up adherence with any exercise delivery model. Moreover, little is known about factors predicting long term adherence; such information can be used in enhancing longer-term exercise programming effects. Hence, the goals of this study are a better understanding of health benefits (QOL, physical fitness, and other clinically relevant endpoints) provided through three varying exercise delivery models that include evaluation of the cost-effectiveness of program provision (for men on ADT for PC), and adherence associated with each approach (during and after the formal six-month intervention period).

The current study is designed to address important limitations in prior studies and advance the science of exercise delivery for men on ADT. First, this study is directly comparing the three main exercise delivery models to identify whether less resource-intense approaches are as efficacious as the reference standard of supervised personal training. Second, adherence will be carefully studied during and, more importantly, after the formal six-month intervention period. Moreover, insights will be gained into enabling factors and barriers to exercise adherence. Third, we will formally begin to evaluate the cost effectiveness of each exercise delivery model; such information should ultimately inform health policy. Additional innovations include structured exercise tapering (to facilitate independent exercise), introduction of a smartphone app and health coach support to enhance home-based exercising, and other features.

The evidence-based knowledge gained with this research will provide an understanding of the best (clinically effective, high adherence, cost effective) exercise program for this population and will help strengthen research in this field. Although the results from the phase II trial will not be definitive, this step is foundational and the knowledge that will be generated will be incorporated into the design and execution of a multi-center phase III RCT.

\section{Abbreviations}

ACSM: American College of Sport Medicine; ADT: Androgen Deprivation Therapy; AED: Automated external defibrillator; BIA: Bioelectrical impedance analysis; BMD: Bone mineral density; BMI: Body mass index; BP: Blood Pressure; CEP: Certified Exercise Physiologist; CPAFLA: The Canadian Physical Activity, Fitness and Lifestyle Approach; CPATH: Canadian Physical Activity Training for Health; CPR: Cardiopulmonary resuscitation; CSEP: Canadian Society of Exercise Physiology; FACT-F: Functional Assessment of Cancer Therapy - Fatigue; FACT-G: Functional Assessment of Cancer Therapy General; FACT-P: Functional Assessment of Cancer Therapy - Prostate; FITT: Frequency, Intensity, Time, Type; HR: Heart Rate; MVPA: Moderate to Vigorous Physical Activity; PARmed-X: Physical Activity Readiness Medical Examination; PAR-Q: Physical Activity Readiness Questionnaire; PC: Prostate Cancer; PSA: Prostate-specific antigen; QOL: Quality of Life; RCT: Randomized Controlled Trial; RPE: Rated perceived exertion; SF-36: Short Form 36, Medical Outcomes Study Quality of Life questionnaire; WC: Waist circumference. 


\section{Competing interests}

The authors declare that they have no competing interests.

\section{Authors' contributions}

SMHA, DSM, PR, CS, MK, GT, AM, RS, PW, and SNCR contributed to the study design and protocol. SD assisted with further study protocol refinement. SMHA and SNCR are study-site leads at their respective institutions. SD and $\mathrm{MO}$ implement and oversee the protocol and data collection. All authors contributed to the writing and approval of the final manuscript.

\section{Acknowledgements}

The authors would like to thank Prostate Cancer Canada for graciously sponsoring the trial.

\section{Author details}

${ }^{1}$ University Health Network, Toronto, ON M5G 2C4, Canada. ${ }^{2}$ University of Toronto, Toronto, ON M5S 2J7, Canada. ${ }^{3}$ University of Guelph Humber, Toronto, ON M9W 5L7, Canada. ${ }^{4}$ York University, Toronto, ON M3J 1P3, Canada. ${ }^{5}$ University of Ottawa, Ottawa, ON K1N 6N5, Canada. ${ }^{6}$ University of Calgary, Calgary, AB T2N 1N4, Canada. ${ }^{7} T o m$ Baker Cancer Centre, Calgary, AB T2N 4N2, Canada. ${ }^{8}$ Toronto General Hospital, 200 Elizabeth St Room EN14-214, Toronto, ON M5G 2C4, Canada.

\section{Received: 24 October 2014 Accepted: 13 April 2015 \\ Published online: 25 April 2015}

\section{References}

1. Canadian Cancer Society's Advisory Committee on Cancer Statistics. Canadian Cancer Society: Canadian Cancer Statistics 2014. Toronto, 2014

2. Siegel R, Ma J, Zou Z, Jemal A. Cancer statistics, 2014. CA Cancer J Clin. 2014;64(1):9-29.

3. Shahinian VB, Kuo YF, Freeman JL, Orihuela E, Goodwin JS. Increasing use of gonadotropin-releasing hormone agonists for the treatment of localized prostate carcinoma. Cancer. 2005;103(8):1615-24

4. Cooperberg MR, Grossfeld GD, Lubeck DP, Carroll PR. National practice patterns and time trends in androgen ablation for localized prostate cancer. J Natl Cancer Inst. 2003:95(13):981-9.

5. Sharifi N, Gulley JL, Dahut WL. Androgen deprivation therapy for prostate cancer. JAMA. 2005:294(2):238-44.

6. Alibhai SMH, Breunis H, Timilshina N, Johnston C, Tomlinson G, Tannock I, et al. Impact of androgen-deprivation therapy on physical function and quality of life in men with non-metastatic prostate cancer. J Clin Oncol. 2010;28(34):5038-45.

7. Alibhai SM, Gogov S, Allibhai Z. Long-term side effects of androgen deprivation therapy in men with non-metastatic prostate cancer: a systematic literature review. Crit Rev Oncol Hematol. 2006;60(3):201-15.

8. Walker LM, Tran S, Robinson JW. Luteinizing hormone-releasing hormone agonists: a quick reference for prevalence rates of potential adverse effects. Clin Genitourin Canc. 2013;11(4):375-84.

9. Baumann FT, Zopf EM, Bloch W. Clinical exercise interventions in prostate cancer patients-a systematic review of randomized controlled trials. Support Care Cancer. 2012;20(2):221-33.

10. Segal RJ, Reid RD, Courneya KS, Malone SC, Parliament MB, Scott CG, et al. Resistance exercise in men receiving androgen deprivation therapy for prostate cancer. J Clin Oncol. 2003;21(9):1653-9.

11. Galvao DA, Nosaka K, Taaffe DR, Spry N, Kristjanson L, McGuigan MR, et al. Resistance training and reduction of treatment side effects in prostate cancer patients. Med Sci Sports Exerc. 2006;38(12):2045-52.

12. Galvao DA, Taaffe DR, Spry N, Joseph D, Newton RU. Combined resistance and aerobic exercise program reverses muscle loss in men undergoing androgen suppression therapy for prostate cancer without bone metastases: a randomized controlled trial. J Clin Oncol. 2010;28(2):340-7.

13. Thorsen L, Courneya KS, Stevinson C, Fossa SD. A systematic review of physical activity in prostate cancer survivors: outcomes, prevalence, and determinants. Support Care Cancer. 2008;16(9):987-97.

14. Santa Mina D, Ritvo P, Segal R, Culos-Reed SN, Alibhai SMH. Exercise After Prostate Cancer Diagnosis. In: Saxton JM, Daley A, editors. Exercise and Cancer Survivorship: Impact on Health Outcomes and Quality of Life. New York: Springer; 2010. p. 113-40.

15. Keogh JW, MacLeod RD. Body composition, physical fitness, functional performance, quality of life, and fatigue benefits of exercise for prostate cancer patients: a systematic review. J Pain Symptom Manage. 2012;43(1):96-110

16. Gardner JR, Livingston PM, Fraser SF. Effects of exercise on treatmentrelated adverse effects for patients with prostate cancer receiving androgen-deprivation therapy: a systematic review. J Clin Oncol. 2014:32(4):335-46.

17. Borst SE. Interventions for sarcopenia and muscle weakness in older people. Age Ageing. 2004;33(6):548-55.

18. Santa Mina D, Ritvo P, Matthew AG, Rampersad A, Stein H, Cheung AM, et al. Group exercise versus personal training for prostate cancer patients: a pilot randomized trial. J Canc Ther. 2012;3(2):146-56.

19. Hansen PA, Dechet CB, Porucznik CA, LaStayo PC. Comparing eccentric resistance exercise in prostate cancer survivors on and off hormone therapy: a pilot study. Phys Rehabil Med. 2009;1(11):1019-24.

20. Bourke L, Doll H, Crank H, Daley A, Rosario D, Saxton JM. Lifestyle intervention in men with advanced prostate cancer receiving androgen suppression therapy: a feasibility study. Canc Epidemiol Biomarkers Prev. 2011;20(4):647-57.

21. Culos-Reed SN, Robinson JL, Lau H, O'Connor K, Keats MR. Benefits of a physical activity intervention for men with prostate cancer. J Sport Exerc Psychol. 2007:29(1):118-27.

22. Culos-Reed SN, Robinson JW, Lau H, Stephenson L, Keats M, Norris S, et al. Physical activity for Men receiving androgen deprivation therapy for prostate cancer: benefits from a 16-week intervention. Support Care Cancer. 2010;18(5):591-9.

23. Windsor PM, Nicol KF, Potter J. A randomized, controlled trial of aerobic exercise for treatment-related fatigue in men receiving radical external beam radiotherapy for localized prostate carcinoma. Cancer. 2004;101(3):550-7.

24. Canadian Society of Exercise Physiology. Physical Activity Readiness Questionnaire. Ottawa: Canadian Society of Exercise Physiology; 2002.

25. Galvao DA, Taaffe DR, Spry N, Joseph D, Newton RU. Acute versus chronic exposure to androgen suppression for prostate cancer: impact on the exercise response. J Urol. 2011;186(4):1291-7.

26. Canadian Society of Exercise Physiology. Physical Activity Training for Health. Ottawa: Health Canada; 2013.

27. Canadian Society of Exercise Physiology. The Canadian Physical Activity, Fitness \& Lifestyle Approach. 3rd ed. Ottawa: Health Canada; 2010.

28. American College of Sports Medicine. ACSM's Guidelines for Exercise Testing and Prescription. New York: Lippincott Williams and Winkins; 2005.

29. Strassmann A, Steurer-Stey C, Lana KD, Zoller M, Turk AJ, Suter P, et al. Population-based reference values for the 1-min sit-to-stand test. Int J Publ Health. 2013;58(6):949-53.

30. Bohannon RW. Sit-to-stand test for measuring performance of lower extremity muscles. Percept Mot Skills. 1995;80(1):163-6.

31. Mathiowetz V, Kashman N, Volland G, Weber K, Dowe M, Rogers S. Grip and pinch strength: normative data for adults. Arch Phys Med Rehabil. 1985;66(2):69-74.

32. Cella DF, Tulsky DS, Gray G, Sarafian B, Linn E, Bonomi A, et al. The functional assessment of cancer therapy scale: development and validation of the general measure. J Clin Oncol. 1993;11(3):570-9.

33. Esper P, Mo F, Chodak G, Sinner M, Cella D, Pienta KJ. Measuring quality of life in men with prostate cancer using the functional assessment of cancer therapy-prostate instrument. Urology. 1997;50(6):920-8.

34. Cella D. The functional assessment of cancer therapy-anemia (FACT-an) scale: a new tool for the assessment of outcomes in cancer anemia and fatigue. Semin Hematol. 1997:34(3 Suppl 2):13-9.

35. Alibhai SM, Duong-Hua M, Sutradhar R, Fleshner NE, Warde P, Cheung AM, et al. Impact of androgen deprivation therapy on cardiovascular disease and diabetes. J Clin Oncol. 2009;27(21):3452-8.

36. Saylor PJ, Smith MR. Metabolic complications of androgen deprivation therapy for prostate cancer. J Urol. 2009;181(5):1998-2006. discussion 2007-1998.

37. Knowler WC, Barrett-Connor E, Fowler SE, Hamman RF, Lachin JM, Walke EA, et al. Reduction in the incidence of type 2 diabetes with lifestyle intervention or metformin. N Engl J Med. 2002;346(6):393-403.

38. Kraemer WJ, Ratamess NA. Hormonal responses and adaptations to resistance exercise and training. Sports Med. 2005;35(4):339-61.

39. Segal RJ, Reid RD, Courneya KS, Sigal RJ, Kenny GP, Prud'Homme DG, et al. Randomized controlled trial of resistance or aerobic exercise in men receiving radiation therapy for prostate cancer. J Clin Oncol. 2009;27(3):344-51. 
40. Prince SA, Adamo KB, Hamel ME, Hardt J, Connor Gorber S, Tremblay M. A comparison of direct versus self-report measures for assessing physical activity in adults: a systematic review. Int J Behav Nutr Phys Act. 2008;5:56.

41. Canadian Society of Exercise Physiology. Canadian Physical Activity Guidelines. Ottawa: Health Canada; 2011.

42. Freedson PS, Melanson E, Sirard J. Calibration of the Computer Science and Applications, Inc. accelerometer. Med Sci Sports Exerc. 1998;30(5):777-81.

43. Tudor-Locke C, Craig CL, Aoyagi Y, Bell RC, Croteau KA, De Bourdeaudhuij I, et al. How many steps/day are enough? For older adults and special populations. Int J Behav Nutr Phys Act. 2011;8:80.

44. Bronfenbrenner U. Environments in developmental perspective: Theoretica and operational models. In: Friedmand SL, Wachs TD, editors. Measuring Environment Across the Life Span. Washington, D.C: American Psychological Association; 1999. p. 3-28.

45. Cerin E, Saelens BE, Sallis JF, Frank LD. Neighborhood Environment Walkability Scale: validity and development of a short form. Med Sci Sports Exerc. 2006;38(9):1682-91.

46. Williams GC, Grow VM, Freedman ZR, Ryan RM, Deci EL. Motivational predictors of weight loss and weight-loss maintenance. J Pers Soc Psychol. 1996;70(1):115-26.

47. Wilson PM, Garcia Bengoechea E. The relatedness to others in physical activity scale: evidence for structural and criterion validity. J Appl Biobehav Res. 2010;15(2):61-87.

48. Markland DA, Tobin V. A modification to the behavioural regulation in exercise questionnaire to include an assessment of amotivation. J Sport Exerc Psychol. 2004;26:191-6.

49. Brunet J, Sabiston CM. Exploring motivation for physical activity across the adult lifespan. Psychol Sport Exerc. 2011;12:99-105.

50. Courneya KS, Friedenreich CM. Utility of the theory of planned behavior for understanding exercise during breast cancer treatment. Psychooncology. 1999;8(2):112-22.

51. Courneya KS, Vallance JK, Culos-Reed SN, McNeely ML, Bell GJ, Mackey JR, et al. The Alberta moving beyond breast cancer (AMBER) cohort study: a prospective study of physical activity and health-related fitness in breast cancer survivors. BMC Cancer. 2012;12:525.

52. Borg GA. Psychophysical bases of perceived exertion. Med Sci Sports Exerc. 1982;14(5):377-81.

53. Heyward VH. Advanced Fitness Assessment and Exercise Prescription. 5th ed. Windsor: Human Kinetics Publishers; 2010.

54. Common Terminology Criteria for Adverse Events (CTCAE) version 4.0. U.S. Department of Health and Human Services, National Institutes of Health, National Cancer Institute. 2009. http://ctep.cancer.gov/protocolDevelopment/ electronic_applications/ctc.htm\#ctc_40, accessed April 22, 2015.

55. Roset M, Badia X, Mayo NE. Sample size calculations in studies using the EuroQol 5D. Qual Life Res. 1999;8(6):539-49.

56. Krahn M, Bremner KE, Tomlinson G, Ritvo P, Irvine J, Naglie G. Responsiveness of disease-specific and generic utility instruments in prostate cancer patients. Qual Life Res. 2007;16(3):509-22.

57. Krahn M, Ritvo P, Irvine J, Tomlinson G, Bremner KE, Bezjak A, et al. Patient and community preferences for outcomes in prostate cancer: implications for clinical policy. Med Care. 2003;41(1):153-64.

58. Thabane L, Ma J, Chu R, Cheng J, Ismaila A, Rios LP, et al. A tutorial on pilot studies: the what, why and how. BMC Med Res Methodol. 2010;10:1.

59. Hertzog MA. Considerations in determining sample size for pilot studies. Res Nurs Health. 2008;31(2):180-91.

60. Sim J, Lewis $M$. The size of a pilot study for a clinical trial should be calculated in relation to considerations of precision and efficiency. J Clin Epidemiol. 2012;65(3):301-8.

61. Demark-Wahnefried W, Clipp EC, Lipkus IM, Lobach D, Snyder DC, Sloane R, et al. Main outcomes of the FRESH START trial: a sequentially tailored, diet and exercise mailed print intervention among breast and prostate cancer survivors. J Clin Oncol. 2007;25(19):2709-18.

62. Richman EL, Kenfield SA, Stampfer MJ, Paciorek A, Carroll PR, Chan JM. Physical activity after diagnosis and risk of prostate cancer progression: data from the cancer of the prostate strategic urologic research endeavor. Cancer Res. 2011;71(11):3889-95.

\section{Submit your next manuscript to BioMed Central and take full advantage of:}

- Convenient online submission

- Thorough peer review

- No space constraints or color figure charges

- Immediate publication on acceptance

- Inclusion in PubMed, CAS, Scopus and Google Scholar

- Research which is freely available for redistribution

Submit your manuscript at www.biomedcentral.com/submit 